\title{
La educación para la salud en la adolescencia temprana para afrontar los cambios físicos y emocionales 1
}

Institución: Universidad de Costa Rica, Maestría en Enfermería Pediátrica

\section{RESUMEN}

La etapa de la adolescencia se caracteriza por gran cantidad de cambios que se concentran en diferentes áreas, como la física, psicológica y emocional, según las subetapas que vayan afrontando. La educación en salud para el adolescente constituye una problemática para la atención pública y un desafío para la actuación de la enfermería, principalmente porque se trata de una etapa de la vida marcada por intensas transformaciones y grandes influencias socioculturales que perduran desde la pubertad hasta la vida adulta. Un acompañamiento apropiado para la edad, por parte de profesionales capacitados, es la clave para reducir factores de riesgo de la etapa para que los adolescentes se sientan seguros de sí y de la forma en que afrontarlos. El objetivo de esta revisión breve es analizar la mejor evidencia científica disponible respecto de la educación para la salud respecto de los cambios en la adolescencia temprana, impartida por enfermería para mejorar el afrontamiento del adolescente ante dichos cambios. Se utilizó como metodología la práctica clínica basada en la evidencia: inicialmente, se planteó una pregunta clínica de tipo pronóstico, así como objetivos acordes a esta, con base en los que se identificó un conjunto de descriptores a partir de los que se realizó la búsqueda de evidencia científica en diferentes bases de datos.

Palabras claves: adolescencia-temprana, cambios-bioemocionales, educación-para-la-salud

\footnotetext{
${ }^{1}$ Fecha de recepción: 5 de diciembre 2017

Fecha de aceptación: 23 de marzo del 2018

${ }^{2}$ Enfermera, estudiante de maestría en Enfermería Pediátrica con énfasis en niñez y adolescencia. Universidad de Costa Rica.

OstoCareCr. Costa Rica. Correo electrónico: majofernandez7@gmail.com

${ }^{3}$ Enfermera, estudiante de maestría en Enfermería Pediátrica con énfasis en niñez y adolescencia. Universidad de Costa Rica. Hospital

Nacional de Niños. Costa Rica. Correo electrónico: vamari83@gmail.com
} 


\title{
Education for health in early adolescence to face the changes physical and emotional ${ }^{1}$
}

Institution: University of Costa Rica, Postgraduate Pediatric Nursing

\author{
María José Fernández Arias ${ }^{2}$ \\ Vanessa Marín Sanabria ${ }^{3}$
}

\begin{abstract}
The stage of adolescence is known to involve a lot of changes; these are concentrated in different areas from physical to psychological and emotional according to the sub-steps that they face. Health education for adolescents is a problem for public attention and a challenge for the performance of nursing, mainly due to the fact that adolescence is a stage of life marked by intense physical, emotional and great sociocultural transformations that They last from puberty to adult life. Age-appropriate accompaniment by trained professionals is the key to reducing risk factors for the stage and for children to feel confident about themselves and the way they are going to face them. The objective of this brief review is to analyze the best available scientific evidence in relation to health education regarding changes in early adolescence, taught by nursing to improve the adolescent's coping with these changes. The methodology recommended for Clinical Practice based on evidence was used as a methodology, initially a prognostic type clinical question was posed and the objectives according to this were raised, based on which a set of descriptors was identified from the that the search for scientific evidence was made in different scientific databases.iptors was identified from which it was made the search for scientific evidence in different scientific databases.
\end{abstract}

Keywords: adolescence-early, changes-bio-emotional, education-for-health

\footnotetext{
${ }^{1}$ Date of receipt: December 5, 2017

Date of acceptance: March 23, 2018

${ }^{2}$ Nurse, Master's student in Pediatric Nursing with emphasis on Childhood and Adolescence. University of Costa Rica. OstoCareCr. Costa Rica. E-mail: majofernandez7@,gmail.com

${ }^{3}$ Nurse, Master's student in Pediatric Nursing with an emphasis on Childhood and Adolescence. University of Costa Rica. National Children's Hospital. Costa Rica. E-mail: vamari83@gmail.com
} 


\title{
Educação para a saúde no início da adolescência para enfrentar as mudanças físico e emocional ${ }^{1}$
}

\author{
María José Fernández Arias ${ }^{2}$ \\ Vanessa Marín Sanabria ${ }^{3}$
}

Instituição: Universidade da Costa Rica, Enfermagem pediátrica de pós-graduação

\section{RESUMO}

Sabe-se que o estágio da adolescência envolve muitas mudanças, que se concentram em diferentes áreas de física a psicológica e emocional de acordo com os sub-passos que enfrentam. A educação em saúde para adolescentes é um problema para a atenção do público e um desafio para o desempenho da enfermagem, principalmente devido ao fato de a adolescência ser um estágio de vida marcado por intensas transformações socioculturais físicas, emocionais e grandes que Eles duram da puberdade para a vida adulta. $\mathrm{O}$ acompanhamento apropriado para a idade por profissionais treinados é a chave para reduzir os fatores de risco do palco e para que as crianças se sintam confiantes em si mesmas e na maneira como elas vão enfrentá-las. O objetivo desta breve revisão é analisar as melhores evidências científicas disponíveis em relação à educação em saúde em relação às mudanças no início da adolescência, ensinadas por enfermagem para melhorar o enfrentamento do adolescente com essas mudanças. A metodologia recomendada para a prática clínica baseada em evidências foi utilizada como metodologia, inicialmente foi colocada uma questão clínica do tipo prognóstico e os objetivos de acordo com ela foram estabelecidos, com base em quais um conjunto de descritores foi identificado a partir da que a busca de evidências científicas foi feita em diferentes bases de dados científicas.

Palavras-chave: adolescência-cedo, mudanças-bio-emocional, educação-para-a-saúde

\footnotetext{
${ }^{1}$ Data de recepção: 5 de dezembro de 2017

Data de aceitação: 23 de março de 2018 ${ }^{2}$ Enfermeira. Mestre em Enfermagem Pediátrica com ênfase na Infância e na Adolescência. Universidade da Costa Rica OstoCareCr. Costa Rica. Correio eletrônico: majofernandez7@gmail.com

${ }^{3}$ Enfermeira, aluna de mestrado em enfermagem pediátrica com ênfase na Infância e na Adolescência. Universidade da Costa Rica Hospital Nacional da Criança. Costa Rica. Correio eletrônico: vamari83@gmail.com
} 


\section{Revista Electrónica Enfermeria Actual en costa Rica}

\section{INTRODUCCIÓN}

La etapa de la adolescencia se caracteriza por gran cantidad de cambios que se concentran en diferentes áreas, como la física, psicológica y emocional, según las subetapas que vayan afrontando. En este caso, el punto de concentración es la adolescencia temprana en la que los jóvenes inician cambios que, muchas veces, ocasionan ansiedad por lo desconocido, miedo a los cambios que presenta su cuerpo y las nuevas responsabilidades que afloran. Por lo anterior, $\mathrm{n}$ acompañamiento apropiado para la edad, y por parte de profesionales capacitados, es la clave para reducir las ansiedades de la etapa y que los menores se sientan seguros de sí y de cómo afrontar su nueva realidad.

En el 2013, Martínez, Alvarado y Campos ${ }^{1}$ mencionaron que es importante que en cualquier abordaje e intervención que se realice con la población adolescente se tome en cuenta cada factor que influye en sus características individuales como de grupo, motivo por el que, el acercamiento a los adolescentes desde enfermería, debe hacerse de una manera integral tomando en cuenta no solo los aspectos biológicos, sino todos aquellos elementos que influyen directamente en el pensamiento y estilo de vida de ese grupo, lo cual incluye familia, redes de apoyo, aspectos psicosociales, alimentación, sexualidad, oportunidades que pueda ofrecer la comunidad en la que se desarrolla, accesibilidad a servicios básicos y servicios de salud, entre otros determinantes sociales que son ejes fundamentales en el desarrollo físico, psicológico y social.

Respecto de la educación para la salud de parte de enfermería, en Educación para la salud integral del adolescente a través de promotores pares, Blanco ${ }^{2}$ menciona que hablar de adolescencia es referirse a transformaciones y de cambios y a nuevas formas de mirar y vivir el mundo. Para muchas personas es hablar de conflicto, de peligro, de rebeldía, de delincuencia y de enfermedad; para otras, de potencialidad, desarrollo, posibilidad para conformar valores como libertad, autodeterminación, responsabilidad, equidad, solidaridad, respeto, no violencia y autocuidado. Además, menciona que la promoción de la salud integral del adolescente se basa en fortalecer los factores de protección y prevenir los de riesgo que modifican positiva o negativamente las características que definen la adolescencia como la búsqueda de la identidad y la independencia, así como el desarrollo de la creatividad, sensibilidad, proyecto de vida, sexualidad y la educación. Lo anterior expresa la importancia de la intervención de enfermería y presencia durante el proceso de desarrollo de esta etapa del ser humano tan vulnerable.

En otro artículo relacionado con la educación de parte de enfermería en adolescentes, Mendes ${ }^{3}$ afirma que la educación en salud para el adolescente tiene el propósito de estimular el autocuidado a partir de hábitos saludables de vida. En lo que se refiere a la prevención del uso y abuso de drogas, la divulgación de la salud del adolescente se constituye en una problemática para la atención pública y un desafío para la actuación de la enfermería, principalmente porque la adolescencia es una etapa de la vida marcada por intensas transformaciones físicas, emocionales y grandes influencias socioculturales que perduran desde la pubertad hasta la vida adulta.

Por su parte, Lago, Tizón, Porto, Fernández ${ }^{4}$ en el año 2014, realizaron una investigación en la que mencionan que, como profesionales sanitarios, la función como educadores no solo se debe limitar a la consulta, a la seguridad y superioridad que genera estar entre cuatro paredes, sino que hay que ir mucho más allá: es necesario salir a la calle y enfrentar la realidad del adolescente, con su contexto sociocultural, acercarse a estos, 


\section{Revista Electrónica Enfermeria Actual en costa Rica}

tratándolos como iguales y comprendiéndolos, con empatía, para ofrecerles unos conocimientos personalizados, realistas y que aborden realmente los aspectos que les preocupan y motivan. Las clases magistrales o las charlas unidireccionales solo sirven para acumular información que difícilmente llegará a modificar sus conductas, ya que el no sentirse identificados o motivados hará que dicha información se quede en simples teorías. Los alumnos deben poder expresar sus miedos y dudas, interaccionar y ser ellos los que vayan elaborando su cuerpo de conocimientos, para lo que se les debe brindar las herramientas necesarias para que sustenten sus propias decisiones, de manera responsable y consecuente con sus acciones. Los programas educativos (de ámbito escolar, sanitario o intersectorial) deben tener en cuenta la participación de los jóvenes como elemento clave para asegurar la eficacia de las intervenciones.

En la Paz, Bolivia en el año 2004, Flores y Díaz ${ }^{5}$ evaluaron el impacto de un programa de capacitación sobre la atención y abordaje a adolescentes para el personal de salud. Para la población adolescente, la relación con profesionales de salud es de vital importancia, a causa de los cambios drásticos, personales y sociales por los cuales atraviesa, los cuales se producen como consecuencia de las conductas tomadas por el adolescente ante dificultades sin resolver; tal es el caso del poco contacto social como resultado de conductas de aislamiento o evitación, que no contribuye con el desempeño de roles sociales ajustados. Las relaciones sociales determinan la efectividad con que el adolescente evalúa y afronta los acontecimientos de su existencia diaria, además de que contribuyen a ajustar la forma de comportarse, de manera que sea recompensado socialmente, mientras adquiere y aprende nuevos comportamientos que generan mayores repertorios conductuales, lo que patentiza la importancia del grupo social para el individuo.

Por otro lado, en Colombia se encontró un análisis del concepto de soledad, el cual brinda una visión de cambio emocional que cursan los adolescentes donde Carvajal-Carrascal, Caro-Castillo en el año $2009^{6}$ menciona que los espacios creados por las enfermeras(os) para la interacción y el cuidado pueden apuntar a disminuir los sentimientos de desesperanza, soledad y sufrimiento que vive el adolescente. Comunicar esperanza y enseñar nuevas formas de afrontamiento también representan herramientas para el trabajo con los jóvenes. El cuidado de enfermería para los adolescentes que sufren de soledad es un proceso interpersonal que se fundamenta en el establecimiento de una atmósfera cálida, que transmita preocupación e interés, y que demuestre empatía por el paciente, su situación y su familia, sumado a otros aspectos importantes en el cuidado como el respeto por sus opiniones y sentimientos, y la protección de la autonomía.

Es importante acotar que la etapa de la adolescencia ha sido abordada de una manera secundaria frente a la sociedad aun cuando, según lo referente a la niñez y adolescencia, en Costa Rica existe un código de la niñez y adolescencia, el cual procura velar por los derechos y deberes de esta población; no obstante, es evidente que se cuenta con poca evidencia de intervenciones adecuadas para el adolescente, una etapa de transición en la que ya no es un niño mas, tampoco, un adulto.

Según la definición de la Organización Mundial para la Salud (OMS) y la Organización Panamericana de la Salud (OPS), la adolescencia es una etapa del ciclo de vida humana cuyo proceso de crecimiento y desarrollo biológico, psicológico, social, creativo y espiritual acontece desde los diez hasta los diecinueve años inclusive. En Costa Rica, la población comprendida entre los 12 y 19 años, según el Instituto Nacional de Estadística y Censos (INEC), está constituida por 725971 personas, lo que significa un 15,7\% de la población total del país, 


\section{Revista Electrónica Enfermeria Actual en costa Rica}

con una distribución porcentual por sexo de 52\% hombres y $58 \%$ mujeres. Sin embargo, se observa que el INEC no incluye en estos datos al sector que se encuentra entre los 10 y los 12 años $^{7}$.

El actual sistema social costarricense experimenta cambios cada vez más acelerados y complejos; por tanto, la mirada a las personas adolescentes demanda un enfoque holístico y sistémico que aborde al ser como unidad multidimensional (física, emocional, mental, social, en el contexto universal y espiritual), porque estos sistemas están interrelacionados y requieren recursos diversos con su adecuada accesibilidad (sociales, materiales, naturales) para posibilitar una adecuada sustentabilidad (BIEN SER Y BIEN ESTAR) de las personas adolescentes en el emergente social. Desde este fundamento, una sociedad en proceso de rápida transición precisa de un paradigma que persiga concentrar acciones conducentes al logro de modificaciones, tanto de forma como de fondo, proyectadas al ser, conocer, hacer y convivir de personas adolescentes, en congruencia con el sistema social imperante y con su participación activa ${ }^{7}$.

En torno al tema, la Asociación Médica Americana recomienda un control de salud anual a adolescentes basado en la multiplicidad y rapidez de los cambios biopsicosociales que ocurren en esta etapa y la necesidad de la identificación precoz de los factores y conductas de riesgo. La supervisión de salud debe ser un esfuerzo compartido entre adolescentes, padres, profesionales de salud, profesores y otros actores involucrados en el proceso de crecimiento y desarrollo del adolescente, máxime que el adolescente concurre poco en los servicios de salud, por ende, cada visita de supervisión de salud debe ser una oportunidad de evaluación clínica integral biopsicosocial y de educación al adolescente y su familia ${ }^{8}$.

Respecto de la adolescencia, se clasifica en tres fases o subetapas de desarrollo psicosocial ${ }^{9}$ que modulan la forma de aproximación, de acogida y en general la relación profesional de salud - adolescente. Estas etapas son

Adolescencia temprana o inicial: entre los 10 y 13 años

Adolescencia media: entre los 14 y 16 años

Adolescencia tardía: entre los 17 y 19 años

Respecto de la educación en salud para el adolescente, tiene el propósito de estimular el autocuidado a partir de hábitos saludables de vida. La divulgación de la salud del adolescente constituye una problemática para la atención pública y un desafío para la actuación de la enfermería, principalmente por el hecho de que la adolescencia es una etapa de la vida marcada por intensas transformaciones físicas, emocionales y grandes influencias socioculturales que perduran desde la pubertad hasta la vida adulta ${ }^{10}$.

Dado lo anterior, es fundamental la detección precoz de los factores de riesgo asociados a la problemática en cuestión que pueden ser sociodemográficos o de orden familiar, inclusive debido a la influencia ejercida por los medios de comunicación, como por ejemplo, cambios de comportamiento, el profesional de la salud podrá analizar y actuar con mejor orientación en la prevención de los problemas ${ }^{10}$. En ese sentido, la educación en el área de la salud se constituye en una importante estrategia para la actuación de la enfermería dentro de este escenario, principalmente en lo que se refiere a implementar programas de atención al adolescente para estimular el autocuidado y adopción de hábitos saludables de vida a través de la valorización del adolescente como sujeto del cuidado y de la atención a la familia y la escuela. 
La importancia de esta revisión breve radica en el valor que se ha otorgado a la divulgación de la salud a través de la educación preventiva, uno de los ejes que fundamentan las acciones del Programa de Salud Del Adolescente $^{11}$. En relación con lo anterior, es fundamental analizar crítica y reflexivamente las acciones de enfermería en la población adolescente, una etapa crucial para el desarrollo humano, orientada a construir futuras personas adultas productivas con un concepto de autocuidado favorable. A lo mencionado, se suma que el ejercer la enfermería basada en la evidencia proporciona mejores intervenciones de enfermería referidas, en este caso, a la promoción de la salud por parte de enfermería al adolescente con el fin de que desarrolle habilidades y capacidades para enfrentar los cambios propios de su etapa de desarrollo e identifique factores de riesgo que alteren su salud integral.

La presente revisión breve tiene como objetivo analizar la mejor evidencia científica disponible en cuanto a la educación para la salud respecto de los cambios en la adolescencia temprana, impartida por enfermería para mejorar el cómo afrontar dichos cambios.

\section{MÉTODO}

Se aplicará la metodología de práctica clínica basada en la evidencia, la cual establece cinco pasos: 1. Establecimiento de una pregunta clínica 2. Búsqueda de la mejor evidencia científica 3. Análisis crítico de la mejor evidencia encontrada 4. Transferencia de la evidencia científica y 5. Evaluación de la transferencia de la evidencia científica.

\section{Pregunta clínica}

La pregunta de revisión es la siguiente: ¿En la adolescencia temprana, la educación para la salud respecto de los cambios en la adolescencia, impartida por enfermería, mejora el cómo afronta el adolescente dichos cambios? Lo anterior se desglosa en el siguiente acrónimo PICO:

\begin{tabular}{|c|l|c|l|}
\hline Población & \multicolumn{1}{|c|}{ Intervención } & Comparación & \multicolumn{1}{c|}{ Resultado (Outcome) } \\
\hline Adolescencia temprana & $\begin{array}{l}\text { La educación para la salud } \\
\text { respecto de los cambios } \\
\text { en la adolescencia } \\
\text { impartida por enfermería }\end{array}$ & $\begin{array}{l}\text { Mejora el afrontamiento } \\
\text { del adolescente a dichos } \\
\text { cambios }\end{array}$ \\
\hline
\end{tabular}

El tipo de pregunta se clasifica como una pregunta de "pronóstico" que utiliza estudios de cohorte longitudinal, para responderla.

\section{Criterios de inclusión}

Población: se considerará estudios que incluyan adolescentes en etapa temprana, independientemente del sexo, edad. 


\section{Revista Electrónica Enfermeria Actual en costa Rica}

Intervención: se considerará estudios que reporten experiencias en educación para la salud -impartida por enfermería- respecto de los cambios en la adolescencia.

Outcome (resultado): se incluirá estudios que establezcan entre sus resultados la educación para la salud en relación con el afrontamiento a los cambios que se suscitan en el adolescente en etapa temprana.

\section{Tipos de estudio}

Se considerará los estudios publicados no experimentales; los diseños incluyen estudios de cohorte longitudinal, cuya publicación esté en idioma español, inglés, portugués.

\section{Estrategia de búsqueda}

La estrategia de búsqueda tendrá como objetivo encontrar estudios científicos publicados, que siguen tres pasos: inicialmente, se llevará a cabo una búsqueda limitada en MedLine, Google Scholar, EBSCO y Cochrane Plus por afinidad con el título de la investigación, además de considerar que el resumen de la investigación contenga los elementos de relevancia claramente expuestos en este apartado, como lo son el objetivo de la investigación, la metodología, así como resultados y conclusiones principales. Luego, se realizará una segunda búsqueda utilizando los descriptores planteados en esta revisión en las bases de datos PubMed, Cochrane, Science Direct, Scielo y Clinical Key. En tercer lugar, se buscará estudios adicionales en la lista de referencias de todos los informes y artículos identificados. Se incluirá toda la información disponible entre el 2013 y el 2017.

\section{Descriptores de búsqueda}

Para la búsqueda de información, se utilizará los descriptores, en idioma español, inglés y portugués expuestos en la tabla 1, empleando AND, OR, NOT.

Tabla 1. Descriptores para la búsqueda de información

\begin{tabular}{|c|c|c|}
\hline \multicolumn{3}{|c|}{ Descriptores } \\
\hline Español & Inglés & Portugués \\
\hline Adolescencia temprana & Early adolescence & início da adolescência \\
\hline $\begin{array}{l}\text { Educación para la salud a los } \\
\text { adolescentes }\end{array}$ & 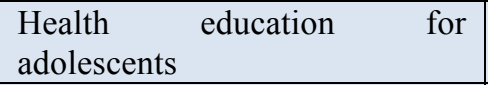 & $\begin{array}{lll}\begin{array}{l}\text { Educação em saúde para } \\
\text { adolescentes }\end{array} & & \text { pala } \\
\end{array}$ \\
\hline $\begin{array}{llll}\begin{array}{l}\text { Cambios físicos } \\
\text { adolescencia }\end{array} & \text { en } & \text { la } \\
\end{array}$ & $\begin{array}{ll}\begin{array}{l}\text { Physical } \\
\text { adolescence }\end{array} & \text { changes } \\
\end{array}$ & $\begin{array}{ll}\begin{array}{l}\text { Mudanças } \\
\text { adolescencia }\end{array} & \text { físicas } \\
\end{array}$ \\
\hline $\begin{array}{l}\text { Cambios emocionales en el } \\
\text { adolescente }\end{array}$ & $\begin{array}{l}\text { Emotional changes in the } \\
\text { adolescent }\end{array}$ & $\begin{array}{ll}\begin{array}{l}\text { Mudanças } \\
\text { adolescencia }\end{array} & \text { emocionais na } \\
\end{array}$ \\
\hline $\begin{array}{l}\text { Educación para la salud por } \\
\text { enfermería }\end{array}$ & Nursing education for health & $\begin{array}{l}\text { Educação de enfermagem para } \\
\text { a saúde }\end{array}$ \\
\hline $\begin{array}{l}\text { Afrontamiento de cambios } \\
\text { físicos y emocionales por el } \\
\text { adolescente }\end{array}$ & $\begin{array}{l}\text { Coping with physical and } \\
\text { emotional changes by the } \\
\text { adolescent }\end{array}$ & $\begin{array}{l}\text { Lidar com mudanças físicas e } \\
\text { emocionais pelo adolescente }\end{array}$ \\
\hline
\end{tabular}

Fuente: elaboración propia 


\section{Selección de los estudios}

Luego de la búsqueda de información, todas las citas identificadas se recopilará y se cargará en el software bibliográfico Mendeley y se eliminará los duplicados. Se examinará los títulos y los resúmenes para evaluarlos según los criterios de inclusión para la revisión. Los estudios que cumplan con los criterios de inclusión se recuperará en su totalidad y se importará sus detalles en una matriz de datos de Excel. El texto completo de los estudios seleccionados se recuperará y evaluará en detalle en relación con los criterios de inclusión. Se excluirá los estudios de texto completo que no cumplan con los criterios de inclusión y se proporcionará los motivos de la exclusión en un apéndice del informe de la revisión final.

Los resultados de la búsqueda se anotará en su totalidad en el informe final y se presentará en un diagrama de flujo PRISMA 2009 en su versión en español.

Cualquier desacuerdo que surja entre los investigadores se resolverá mediante una discusión conjunta.

\section{Extracción de los datos}

Se extraerán los datos de los artículos incluidos en la revisión de forma manual. Los datos incluirán detalles específicos, como año, autor, título, tipo y métodos del estudio, participantes, tipo de intervención, selección de la muestra, resultado principal y secundario de importancia para la pregunta clínica planteada y los objetivos específicos.

Cualquier desacuerdo entre los investigadores se resolverá mediante una discusión conjunta. Se contactará a los autores de los documentos para solicitar datos faltantes o adicionales cuando sea necesario.

\section{Evaluación de la calidad metodológica}

Para evaluar la calidad metodológica de la documentación recuperada, se desarrollará un análisis crítico de la evidencia según lo recomiendan Rojas, Cubero y Leiva ${ }^{12}$.

La calidad de la información y su pertinencia para dar respuesta a la pregunta planteada de la investigación se hará a través de la plataforma de fichas de lectura crítica 2.0 (FLC 2.0) que permite analizar la calidad o validez de los artículos científicos según el nivel bajo, medio o alto.

\section{Síntesis de datos}

Los resultados de esta revisión breve serán sintetizados y presentados en forma de tabla de evidencia y resumen narrativo de acuerdo con los objetivos específicos para su análisis posterior.

\section{Conflicto de intereses}

La autora declara que no tiene conflictos de tipo personal, comercial, académico, político o financiero. 


\section{Agradecimiento}

Las autoras del presente artículo agradecen al programa de investigación por la oportunidad de participar en el proceso de la investigación en enfermería, ya que fortalece nuestra criticidad y permite incursionar en la práctica basada en la evidencia científica.

Agradecemos a las profesoras/ facilitadoras por incentivar a incursionar en la investigación en enfermería, por su guía, apoyo y transmisión de conocimiento en el área.

Finalmente, agradecemos a los funcionarios del SIBDI que nos colaboraron en la guía de búsqueda y recomendaciones de cómo mejorar nuestra revisión bibliográfica.

\section{REFERENCIAS BIBLIOGRÁFICAS}

1. Martínez D, Alvarado J, Campos H, Elizondo K, Esquivel H, Mancía A. Intervención de Enfermería en la adolescencia: experiencia en una Institución de Estudios Secundarios Pública. Rev.Actual en Costa Rica. 2013;24.

2. Blanco ME, Jordán M, Pachón L, Sánchez TB, Medina RE. Educación para la salud integral del adolescente a través de promotores pares. Rev. Med. Electrón. 2011;33(3): 349-359.

3. Rodrigues L, Oliveira ML, Assunção M. Bebida alcohólica en la adolescencia: el cuidado-educación como estrategia de acción de la enfermería. Esc. Anna Nery. 2010;14(1):158-164. http://dx.doi.org/10.1590/S1414-81452010000100023

4. Luces AM, Tizón E, Porto M, Fernández C. La importancia de enfermería en la educación sexual plural durante los primeros años de la adolescencia: rompiendo estereotipos. 2014;8(2). http://dx.doi.org/10.4321/S1988$348 X 2014000200006$.

5. Flores PI, Díaz PA. Evaluation of an upgrading program on the care and approach of adolescents for the health personnel in La Paz, Bolivia. Educ Med Super. 2004; 18(4): 1-1.

6. Carvajal-Carrasca G, Caro-Castillo CV. Soledad en la adolescencia: análisis del concepto. Aquichán. 2009; 9(3): 281296.

7. ASCAJU. Análisis, reflexiones y recomendaciones para la acción. Costa Rica: Asociacón Costarricense de Adolescencia y Juventud: 2013.

8. Santander S, González MI, Ramírez LL, Montero A. Guía práctica de Consejería para Adolescentes y Jóvenes. ORIENTACIONES GENERALES. Dirigida a los equipos de Atención Primaria. Chile: Ministerio de Salud:2011.

9. Zubarew T. (2012). Desarrollo integral del adolescente, evaluación clínica. Universidad Católica de Chile, 3,8,22

10. Ferreira MA, Alvim NAT, Teixeira MLO, Veloso RC. Saberes de adolescentes: estilo de vida e cuidado à saúde. Texto Contexto Enferm. 2007; 16(2): 217-24.

11. Ministério da Saúde (BR). Secretaria de Programas e Projetos, Divisão de Saúde Materno-Infantil. PROSAD - Bases Programáticas. Brasília: 1989. 


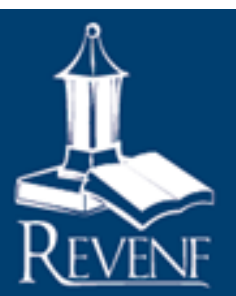

Revista Electrónica Enfermeria Actual en costa Rica

12. Rojas LP, Cubero C, Leiva V. Investigación secundaria: utilización de la mejor evidencia en la práctica clínica. $3^{\mathrm{a}}$. Edición. 2017. San José: CIEBE-CR 